\title{
Proposal for a topological spin Chern pump
}

\author{
C. Q. Zhou, ${ }^{1}$ Y. F. Zhang, ${ }^{1}$ L. Sheng,,${ }^{1, *}$ R. Shen, ${ }^{1}$ D. N. Sheng, ${ }^{2}$ and D. Y. Xing ${ }^{1, \dagger}$ \\ ${ }^{1}$ National Laboratory of Solid State Microstructures and Department of Physics, Nanjing University, Nanjing 210093, China \\ ${ }^{2}$ Department of Physics and Astronomy, California State University, Northridge, California 91330, USA
}

(Received 5 December 2013; published 25 August 2014)

\begin{abstract}
A one-dimensional electron model with parameters modulated adiabatically in closed cycles is proposed which can continuously pump spin to leads. By defining the spin-polarized Wannier functions, we reveal that the spin pumping is protected by the spin Chern numbers, so that it is stable to perturbations violating the time-reversal symmetry and spin conservation. Our work demonstrates the principle for realizing robust topological spin pumps independent of the time-reversal symmetry. A possible way to directly observe the bulk topological invariants is also suggested.
\end{abstract}

DOI: 10.1103/PhysRevB.90.085133

\section{INTRODUCTION}

The integer quantum Hall (IQH) effect discovered in 1980 [1] is the first example of topological state in the field of condensed matter physics. Since then, there has been continuously strong interest in topological phenomena in condensed matter systems. Laughlin [2] interpreted the IQH effect as a quantum charge pump. Increasing the magnetic flux by a single flux quantum that threads a looped IQH ribbon constitutes a cycle of the pump due to gauge invariance, transferring an integer-quantized amount of charge from one edge of the ribbon to the other. Thouless et al. [3] showed that the IQH state can be classified by a topological invariant, the Chern number. Thouless and Niu [4,5] also established a general relation between the Chern number and the charge pumped during a period of slow variation of potential in the Schrödinger equation.

Recently, an important discovery was the topological insulator [6-9], a new quantum state of matter existing in nature. Different from the IQH systems, the topological insulators preserve the time-reversal (TR) symmetry. Two-dimensional topological insulators, also called the quantum spin Hall (QSH) systems, have a bulk band gap and a pair of gapless helical edge states traversing the bulk gap. When electron spin is conserved, the topological properties of the QSH systems can be easily understood, as a QSH system can be viewed as two independent IQH systems without Landau levels [10]. When the spin conservation is destroyed, unconventional topological invariants are needed to classify the QSH systems. The $Z_{2}$ index [11] and the spin Chern numbers [12-14] have been proposed to describe the QSH systems. While the two different invariants are found to be equivalent to each other for TR-invariant systems $[13,14]$, they lead to controversial predictions when the TR symmetry is broken. The definition of the $Z_{2}$ index explicitly relies on the presence of TR symmetry, suggesting that the QSH state turns into a trivial insulator once the TR symmetry is broken. However, calculations [15] based upon the spin Chern numbers showed that the nontrivial topological properties of the QSH systems remain intact when the TR symmetry is broken, as long as the band gap and spin spectrum gap stay open. The nonzero spin Chern numbers

\footnotetext{
*shengli@nju.edu.cn

†dyxing@nju.edu.cn
}

PACS number(s): 72.25.-b, 73.23.-b, 73.43.-f, 75.76.+j guarantee that the edge states must appear on the sample boundary [16], which could be either gapped or gapless, depending on symmetries or spatial distributions of the edge states [17]. This prediction was supported by the recent experimental observation of the QSH effect in InAs/GaSb bilayers under broken TR symmetry [18]. Unlike the Chern number underlying IQH systems, which is embedded into Hall conductivity, the bulk topological invariants in the QSH systems are not directly measurable, though some methods to detect the topological invariants by pumping charge current through the symmetry-protected gapless edge states have been suggested $[19,20]$. These methods are still indirect, in the sense that topological invariants are properties of the bulk electron wave functions.

Spin pumps promise broad applications in spintronics, e.g., the resulting spin battery is the spintronic analog of the charge battery in conventional electronics. While current experimental method practicable at room temperatures was to precess magnets [21,22], several different approaches have been explored, ranging from breaking of spin-reversal symmetry by magnetic field [23-27], rectification of the ac spin current resulting from the Rashba effect [28], to topological spin pumping [29-33]. Besides their fundamental interest, the topological spin pumps [29-33] might be useful for generating low-noise spin currents. When spin $s_{z}$ is conserved, the idea of the Thouless charge pump was extended to construct quantized adiabatic spin pumps [29,30]. However, Fu and Kane argued [31] that, unlike the charge, the spin does not obey a fundamental conservation law, and they introduced a more general concept of the $Z_{2}$ pump. In the $Z_{2}$ pump, while the amount of spin pumped per cycle is not integer quantized, in the absence of spin conservation, the pumping process is protected by a $Z_{2}$ topological invariant, provided that the TR symmetry is present. Meidan et al. [32,33] classified topological pumps based on general properties of the scattering matrix, which is applicable to systems attached to multichannel leads. They showed that in the weak coupling limit, topological spin pumps are characterized by the appearance of symmetry-protected gapless end states during the pumping cycle, similarly to the $Z_{2}$ pump [31]. So far, existing theories seemed to suggest that either spin conservation or TR symmetry is necessary for constructing topological spin pumps, which greatly restricts their practical applications. Robust spin pumps protected by topology alone, independent of any symmetries, are still awaited. 
In this paper, we predict another intriguing effect resulting from the spin Chern numbers, namely, topological spin pumping. A one-dimensional electron model with parameters modulated adiabatically in closed cycles which can continuously pump spin into leads is proposed. By defining the spin-polarized Wannier functions (SPWFs), we reveal that the spin-pumping effect is a direct manifestation of the nontrivial topological properties of the electron wave functions, characterized by nonzero spin Chern numbers. In contrast to the $Z_{2}$ pump, this spin Chern pump remains robust in the presence of magnetic impurities, which destroy both the TR symmetry and spin conservation. Our work demonstrates the possibility and principle to implement topological spin pumps independent of any symmetries. We further show that when a half-metallic lead is connected to the pump, quantized charge $-e(e)$ will be pumped into the lead, for the magnetization of the lead parallel (antiparallel) to the $z$ axis. This suggests an unambiguous way to observe the bulk topological invariants, the spin Chern numbers.

In the next section, we introduce the model Hamiltonian. By defining the SPWFs, we identify nonzero spin Chern numbers as the topological invariants underlying the spin pumping effect in the model. In Sec. III, the spin pumped per cycle is calculated from the scattering matrix formula. In Sec. IV, a comparison between the present theory and some previous theories was made. In Sec. V, a possible way to observe the spin Chern numbers experimentally is introduced. The final section contains a summary.

\section{SPIN CHERN NUMBERS AND WANNIER FUNCTIONS}

We consider a one-dimensional electron model with Hamiltonian [34]

$$
H_{P}=\sum_{\langle i, j\rangle} t_{i, j} c_{i}^{\dagger} c_{j}+g(t) \sum_{i}(-1)^{i} c_{i}^{\dagger} s_{z} c_{i},
$$

where $c_{i}^{\dagger}=\left(c_{i \uparrow}^{\dagger}, c_{i \downarrow}^{\dagger}\right)$ are the creation operators in the spinor representation for electrons with up and down spins on site $i, t_{i, j}$ is the periodically varying hopping integral between the nearest-neighboring sites, given by $t_{i, i+1}=t_{i+1, i}=t_{0}+$ $(-) t_{1} \cos \omega_{0} t$ for $i$ on the $A(B)$ sublattices, $g(t)=g_{0} \sin \omega_{0} t$ is the Zeeman splitting energy, and $s_{z}$ is the Pauli matrix acting on the electron spin. A possible experimental realization of this model is illustrated in Fig. 1. We note that the hopping integral $t_{i, j}$ is modulated both in space and in time with commensurate periods. This modulation can be achieved by stimulating an oscillatory shear deformation of the substrate. It is easy to see that Eq. (1) preserves the TR symmetry, i.e., $H_{P}(-t)=\Theta H_{P}(t) \Theta$, with $\Theta$ as the ordinary TR operator. For an infinitely long chain of atoms, the eigenenergies of Eq. (1) can be obtained by the Fourier transform, yielding

$$
E\left(k_{x}\right)= \pm \sqrt{g^{2}(t)+4 t_{0}^{2} \cos ^{2} \frac{k_{x} a_{0}}{2 \hbar}+\alpha^{2}(t) \sin ^{2} \frac{k_{x} a_{0}}{2 \hbar}},
$$

with $\alpha(t)=2 t_{1} \cos \omega_{0} t$ and $a_{0}$ the lattice constant. Given $t_{0} \gg t_{1}, g_{0}>0$, the system has a middle band gap between $\pm \sqrt{\alpha^{2}(t)+g^{2}(t)}$, which is finite at any time. In the adiabatic limit, on the torus of $k_{x}$ and $t$, one can define the spin Chern numbers $C_{ \pm}$in a standard way [13-15] and obtain $C_{ \pm}= \pm 1$.

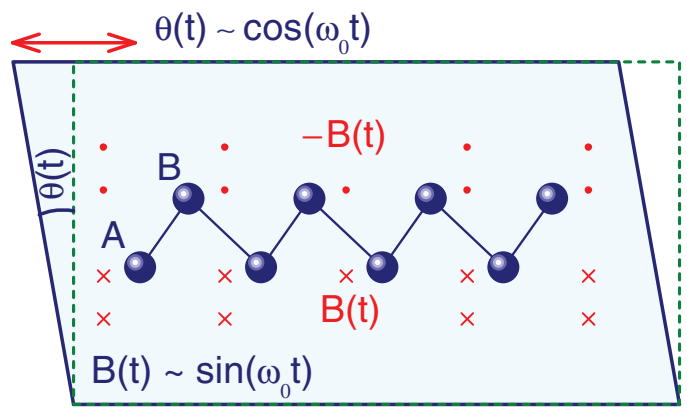

FIG. 1. (Color) A zigzag chain of atoms is affixed on a substrate. Oscillatory shear deformation of the substrate is stimulated, resulting in a periodic modulation of hopping integrals between nearestneighboring atomic sites. A nonuniform oscillating magnetic field is applied in such a manner that electrons on the $A$ and $B$ sites experience opposite Zeeman fields at any time.

In what follows we want to set up a relation of the nontrivial topological properties of the system to the spectral flow of the centers of mass of the SPWFs. In order to show the robustness of nontrivial topological properties, we introduce magnetic impurities with randomly oriented classical spins into the system. The Hamiltonian is given by $H_{I}=V_{0} \sum_{\alpha} c_{\alpha}^{\dagger} \mathbf{s} \cdot \mathbf{m}_{\alpha} c_{\alpha}$, where $\alpha$ runs over all the impurity sites, and $\mathbf{m}_{\alpha}$ is a unit vector in the direction of the $\alpha$ th impurity spin. Apparently, the presence of the magnetic disorder destroys both the spin conservation and TR symmetry of the system.

We diagonalize the total Hamiltonian $H_{P}+H_{I}$ numerically, and the eigenenergies and eigenstates are denoted as $E_{n}$ and $\left|\varphi_{n}\right\rangle$. By using the same procedure as calculating the spin Chern numbers, the occupied valence bands can be partitioned into two spin sectors by diagonalizing the projected spin operator $P s_{z} P$ with $P=\sum_{E_{n}<E_{\mathrm{F}}}\left|\varphi_{n}\right\rangle\left\langle\varphi_{n}\right|$ as the projection operator to the occupied space. If the spin is conserved, the eigenvalues of $P s_{z} P$ have only two values: 1 or -1 . When the spin conservation is broken weakly, there is still a finite gap in the eigenspectrum of $P s_{z} P$, which naturally divides the spectrum into two sectors: spin-up and spin-down sectors. The eigenstates of $P s_{z} P$ for the two spin sectors are denoted by $\left|\psi_{m \pm}\right\rangle$. By definition, $\left|\psi_{m \pm}\right\rangle$ are essentially the maximally spin-polarized states. Then we can construct the Wannier functions $[35,36]\left|\chi_{m \pm}\right\rangle$ for the spin-up and spin-down sectors, respectively, which are called the SPWFs.

The evolution of the centers of mass $\langle x\rangle$ of the SPWFs with time is plotted in Fig. 2 for three different disorder strengths. It is found that in the absence of disorder $\left(V_{0}=0\right)$, all the centers of $\left|\chi_{m+}\right\rangle$ move rightwards, each center on average shifting a lattice constant per cycle, and those of $\left|\chi_{m-}\right\rangle$ move in the opposite direction, as shown in Fig. 2(a). According to the general theory [36], the total displacement of the centers of $\left|\chi_{m+}\right\rangle\left(\left|\chi_{m-}\right\rangle\right)$ per cycle divided by the length of the system is equal to the spin Chern number $C_{+}=1\left(C_{-}=-1\right)$ for the spin-up (spin-down) sector. In the presence of an intermediate strength of magnetic disorder $V_{0}=2.5$, the nontrivial spectral flow of the Wannier centers remains robust, except for small deformations of the flowing paths, as shown in Fig. 2(b). For very strong magnetic disorder, the ordered movement of the Wannier centers is eventually interrupted, although 
o spin up o spin down

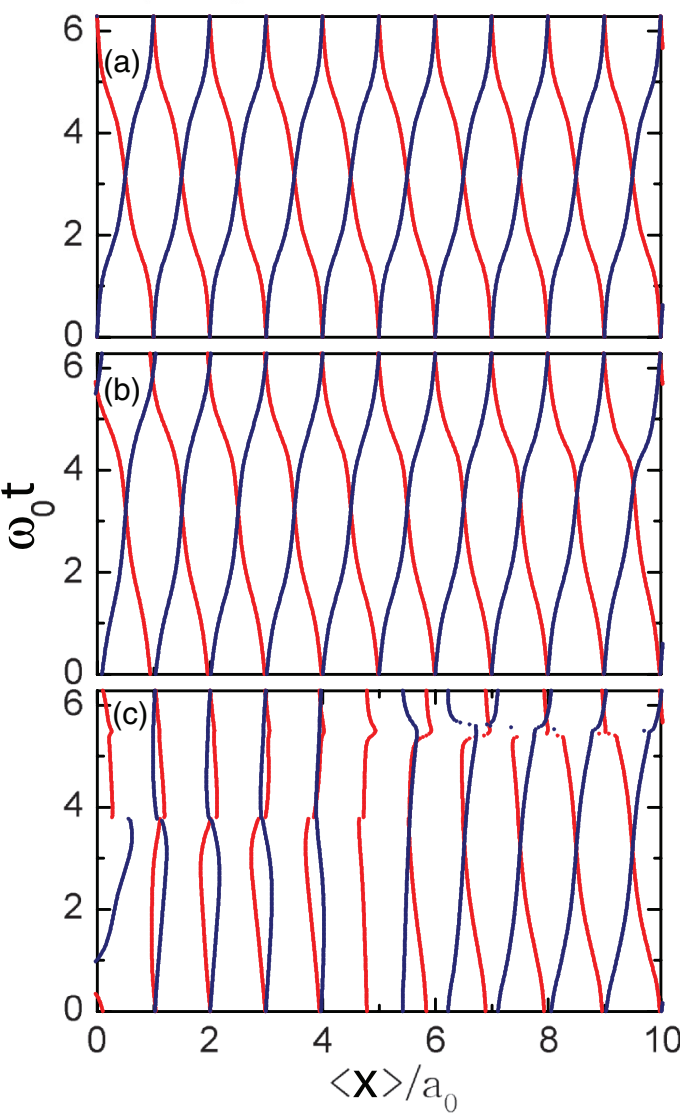

FIG. 2. (Color) Evolution of centers of the Wannier functions for the spin-up and spin-down sectors. For clarity, only ten unit cells of a long chain with periodic boundary condition are displayed, in which $10 \%$ of the atoms are assumed to be replaced with magnetic impurities. The other parameters are taken as $t_{1}=g_{0}=0.1$, and $V_{0}=0$ (a), $V_{0}=2.5(\mathrm{~b})$, and $V_{0}=4.5$ (c), with $t_{0}$ as the unit of energy.

rearrangement of some centers still happens locally, as shown in Fig. 2(c), indicating that the system becomes topologically trivial $\left(C_{ \pm}=0\right)$.

It is worth pointing out that the space spanned by the SPWFs $\left|\chi_{m \pm}\right\rangle$ is identical to that spanned by $\left|\varphi_{n}\right\rangle$ for $E_{n}<E_{\mathrm{F}}$, namely, $\sum_{m}\left|\chi_{m+}\right\rangle\left\langle\chi_{m+}\left|+\sum_{m}\right| \chi_{m-}\right\rangle\left\langle\chi_{m-}\right|=P$. The SPWFs are just another equivalent representation of the occupied space. Therefore, the $N$ electrons occupying the energy eigenstates $\left|\varphi_{n}\right\rangle$ for $E_{n}<E_{\mathrm{F}}$ may also be equivalently considered as two groups: $N / 2$ electrons occupying $\left|\chi_{m+}\right\rangle$ and $N / 2$ electrons occupying $\left|\chi_{m-}\right\rangle$. The counterflows of the centers of the SPWFs observed in Figs. 2(a) and 2(b), as a consequence of the nonzero spin Chern numbers, represent the true movements of the electrons in the spin-up and spin-down sectors with time. Without the TR symmetry and spin conservation, such nontrivial spectral flows become visible only if the occupied space is properly partitioned, as has been done above. It is expected that if leads are strongly connected to the two ends of the atomic chain, the opposite movements of the electrons in the spin-up and spin-down sectors will extend into the leads, transferring spin to the leads continuously. The system becomes a topological spin pump.

\section{CALCULATION OF THE SPIN PUMPED}

We now consider a sufficiently long pump for $x<0$ and a lead for $x>0$, which are in good contact with each other. In order to obtain a transparent analytical expression for the pumped spin, we expand the Hamiltonian equation [Eq. (1)] for the pump around $k=\pi \hbar / a_{0}$, where the band gap is minimal, yielding

$$
H_{P}=\alpha(t) \sigma_{x}+v_{\mathrm{F}} p_{x} \sigma_{y}+g(t) s_{z} \sigma_{z},
$$

where $v_{\mathrm{F}}=t_{0} a_{0} / \hbar, p_{x}=k_{x}-\pi \hbar / a_{0}$, and $\sigma_{x(y, z)}$ are the Pauli matrices associated with the $A B$ sublattices. The Hamiltonian for the lead is taken to be

$$
H_{L}=v_{\mathrm{F}} p_{x} \sigma_{y} .
$$

The Fermi level is set to be $E_{\mathrm{F}}=0$, inside the bulk band gap of the pump. It is assumed that within the decay length of electron wave functions into the pump, there exists only one magnetic impurity sited at $x=0$ with potential $H_{I}=V(x) s_{x}$. In usual cases, when the coupling range of the impurity potential is much smaller than the decay length of the electron wave functions, one can take $V(x)$ to be a $\delta$ function. Unfortunately, it is well known that a $\delta$-like potential barrier is ill-defined for the Dirac electrons. Here, we model $V(x)$ as a square potential centered at $x=0$ with height $V_{0}$ and width $d$. Different assumptions of the shape of $V(x)$ will not change the qualitative conclusion. If $d$ is much smaller than the decay length of wave functions, by taking the $d \rightarrow 0$ limit and keeping $U_{0}=V_{0} d$ finite, it can be shown that the scattering effect of the impurity potential is equivalent to imposing a unitary boundary condition for the electron wave functions:

$$
\Psi\left(x=0^{+}\right)=S \Psi\left(x=0^{-}\right),
$$

where $S=e^{-i \phi \sigma_{y} s_{x}}$ with $\phi=U_{0} / \hbar v_{\mathrm{F}}$. Without the impurity $(\phi=0)$, Eq. (5) will reduce to the ordinary continuity condition $\Psi\left(x=0^{+}\right)=\Psi\left(x=0^{-}\right)$. The impurity potential can also describe a spin-dependent interface potential between the pump and lead. In experiments, such a potential may be induced by putting a ferromagnetic insulating tip in close proximity to the atomic chain.

Calculation of the spin pumped into the lead per cycle amounts to solving the scattering problem of an electron incident at the Fermi level from the lead [37,38]. We first consider the case where the spin of the incident electron is parallel to the $z$ axis. On the bases $(|\uparrow, 1\rangle,|\uparrow,-1\rangle,|\downarrow, 1\rangle$, $|\downarrow,-1\rangle$ ) with the kets as the eigenstates of $s_{z}$ and $\sigma_{z}$, the wave function in the lead is given by

$$
\Psi(x)=\frac{1}{\sqrt{2}}\left(\begin{array}{c}
1 \\
-i \\
0 \\
0
\end{array}\right)+\frac{r_{\uparrow \uparrow}}{\sqrt{2}}\left(\begin{array}{l}
1 \\
i \\
0 \\
0
\end{array}\right)+\frac{r_{\downarrow \uparrow}}{\sqrt{2}}\left(\begin{array}{l}
0 \\
0 \\
1 \\
i
\end{array}\right),
$$

for $x>0$, and that in the pump is given by

$$
\Psi(x)=C_{1}\left(\begin{array}{c}
\sin \frac{\varphi}{2} \\
\cos \frac{\varphi}{2} \\
0 \\
0
\end{array}\right) e^{\gamma x}+C_{2}\left(\begin{array}{c}
0 \\
0 \\
-\sin \frac{\varphi}{2} \\
\cos \frac{\varphi}{2}
\end{array}\right) e^{\gamma x},
$$


for $\quad x<0$. Here, $\varphi=\arg [\alpha(\mathrm{t})+\operatorname{ig}(\mathrm{t})], \quad$ and $\quad \gamma=$ $\sqrt{\alpha^{2}(t)+g^{2}(t)} / \hbar v_{F}$. By substituting Eqs. (6) and (7) into Eq. (5), it is straightforward to derive for the reflection amplitudes $r_{\uparrow \uparrow}=-[\cos (2 \phi) \cos (\varphi)+i \sin (\varphi)]$ and $r_{\downarrow \uparrow}=i \sin (2 \phi) \cos (\varphi)$. Similarly, by considering the case where the spin of the incident electron is antiparallel to the $z$ axis, one can obtain $r_{\downarrow \downarrow}=r_{\uparrow \uparrow}^{*}$ and $r_{\uparrow \downarrow}=r_{\downarrow \uparrow}$.

The $z$ component of the pumped spin per cycle is given by $[37,38]$

$$
\begin{aligned}
\Delta s_{z}= & \frac{\hbar}{4 \pi i} \oint_{T} d t\left(r_{\uparrow \uparrow}^{*} \frac{d r_{\uparrow \uparrow}}{d t}-r_{\downarrow \downarrow}^{*} \frac{d r_{\downarrow \downarrow}}{d t}\right. \\
& \left.-r_{\downarrow \uparrow}^{*} \frac{d r_{\downarrow \uparrow}}{d t}+r_{\uparrow \downarrow}^{*} \frac{d r_{\uparrow \downarrow}}{d t}\right),
\end{aligned}
$$

with $T=2 \pi / \omega_{0}$ as a period of the pump. Here, the third and fourth terms in the integrand have no contribution, since $r_{\uparrow \downarrow}=$ $r_{\downarrow \uparrow}$ is always imaginary. The first and second terms make an equal contribution, due to $r_{\downarrow \downarrow}=r_{\uparrow \uparrow}^{*}$. Therefore, $\Delta s_{z}=$ $\frac{\hbar}{2 \pi i} \oint_{T} r_{\uparrow \uparrow}^{*} d r_{\uparrow \uparrow}$, which can be further evaluated to be

$$
\Delta s_{z}=\frac{\hbar}{2}\left(2-4 \phi^{2}\right)+\mathcal{O}\left(\phi^{4}\right)
$$

for $\phi^{2} \ll 1$. Similarly, one can find $\Delta s_{x}=\Delta s_{y}=0$. In Eq. (9), $\Delta s_{z}$ in units of $\hbar / 2$ is quantized to 2 at $\phi=0$, and there is a small deviation from the quantized value for small $\phi$. This result is consistent with the analysis of the spectral flows of the SPWFs. The small deviation arises from the destruction of the spin conservation by the magnetic impurity, rather than the breaking of the TR symmetry. Such a deviation occurs as well in the absence of the magnetic impurity, if the Rashba spin-orbit coupling is included [31], which destroys the spin conservation but preserves the TR symmetry. Physically, it is because the electron wave function $\Psi(x)$, given by Eqs. (6) and (7), is not an eigenstate of $s_{z}$ when $\phi \neq 0$. Moreover, the direction of the spin polarization of the wave function varies with time ( $\varphi$ is a function of $t)$, and so the quantized value cannot be recovered by properly choosing the spin quantization axis.

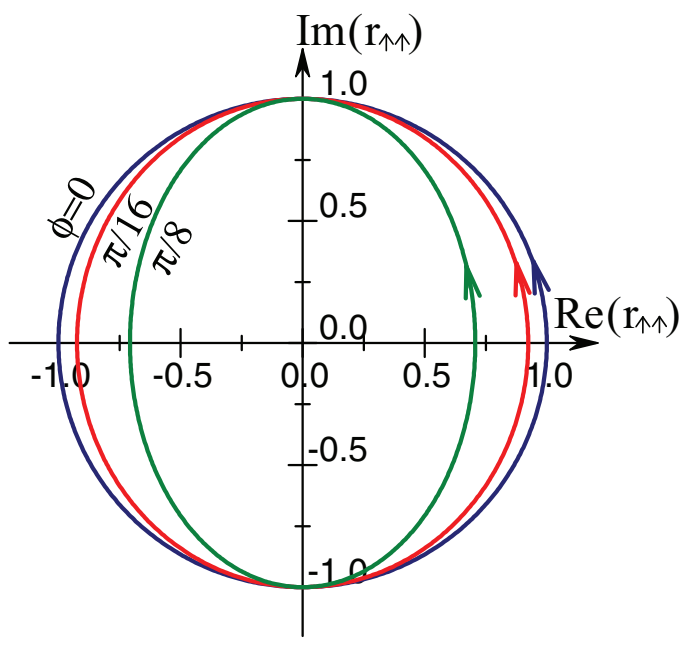

FIG. 3. (Color) Trajectories of $r_{\uparrow \uparrow}$ on the complex plane for three different strengths of impurity scattering potential.
To get some more insight into the spin pump, we plot the trajectories of $r_{\uparrow \uparrow}$ in a cycle on the complex plane in Fig. 3 for three different $\phi$. Each trajectory is a closed orbit simply because the Hamiltonian is periodic in time. At $\phi=0$, the orbit of $r_{\uparrow \uparrow}$ is a unit circle, and increasing $\phi$ deforms the orbit. In general, one can find that $\Delta s_{z}$ equals the area enclosed by the trajectory of $r_{\uparrow \uparrow}$ multiplied by $\hbar / \pi$. Therefore, any small perturbation may cause a small deformation of the trajectory from the unit circle, but can neither stop the spin pumping nor change the sign of $\Delta s_{z}$. This reflects the topological stability of the spin pump from another aspect.

\section{A COMPARISON WITH PREVIOUS THEORIES}

We wish to make a comment on the $Z_{2}$ spin pump proposed by Fu and Kane [31]. These authors studied the same model as Eq. (1), and showed that for a system with closed ends, there is a pair of bound states localized near each end with energy levels crossing the bulk energy gap with time going on. The end states exhibit level crossing at the TR invariant point $t=T / 2$, forming a Kramers doublet. For the system "weakly" coupled to leads, there occurs a resonance in reflection amplitudes, when the Kramers degenerate end states appear. Such a resonance structure allows spin to be pumped into the leads. However, if the TR symmetry is broken, an energy gap will open at the level-crossing point, and the spin pumping will be stopped. Therefore, they concluded that the TR symmetry plays a crucial role in the $Z_{2}$ spin pump. Apparently, the $Z_{2}$ pump essentially reveals the properties of the end states appearing in a closed system.

Meidan et al. [32,33] established a classification of topological pumps based on general properties of the scattering matrix, which is applicable to systems attached to multichannel leads. They showed that in the weak coupling limit, topological spin pumps are characterized by the appearance of TRsymmetry-protected gapless end states during the pumping cycle, similarly to the $Z_{2}$ pump [31]. In general, when the spin conservation is destroyed, the quantization of spin pumped per cycle is not ascertained [31,33]. Interestingly, these authors showed that in the limit of infinitely weak coupling and an infinitely long pumping period, the quantization of spin pumped becomes asymptotically exact $[32,33]$. While such a limit is not reachable in practice, it suggests a direction for optimization of a topological spin pump [32]. Similar to the $Z_{2}$ theory [31] of topological spin pumps, these theories [32,33] are not applicable to systems without TR symmetry.

In contrast to the above theories, here we considered an open system with a "finite" connection between the pump and lead, which allows the electrons to move freely between the pump and leads without the appearance and participation of the end states. We showed that the topological spin pumping remains robust in the presence of TR-symmetry-breaking perturbations. For this reason, the spin pumping process can no longer be explained within the framework of the $Z_{2}$ theory [31] or associated with the appearance of gapless end states [31-33]. Instead, we have demonstrated that it is related to the spin Chern numbers. Therefore, our work identified a more general mechanism that can give rise to topological spin pumping, independent of the TR symmetry. 


\section{OBSERVATION OF SPIN CHERN NUMBERS}

Now we propose a method to experimentally observe the spin Chern numbers by using a half-metallic lead. A half-metal, e.g., $\mathrm{CrO}_{2}, \mathrm{La}_{2 / 3} \mathrm{Sr}_{1 / 3} \mathrm{MnO}_{3}$, etc., is a material that acts as a conductor to electrons of one spin orientation but as an insulator to those of the other spin orientation. From the viewpoint of the electronic structure, the energy band for electron spin parallel to the spin polarization of the half-metal is metallic, whereas the Fermi level falls into an energy gap for electron spin antiparallel to the spin polarization. To model a half-metallic lead, we introduce an additional term $V_{0}\left(1 \pm s_{z}\right) \sigma_{z} / 2$ into the Hamiltonian Eq. (4) of the lead, where \pm is taken, respectively, for the spin polarization of the lead parallel and antiparallel to the $z$ axis. This term opens an energy gap of size $2 V_{0}$ around the Fermi energy for electron spin antiparallel to the spin polarization, without affecting electrons with spin parallel to the spin polarization.

We first consider the case in which the spin polarization is parallel to the $z$ axis so that the spin-down channel is blocked. The wave function in the lead becomes

$$
\Psi(x)=\frac{1}{\sqrt{2}}\left(\begin{array}{c}
1 \\
-i \\
0 \\
0
\end{array}\right)+\frac{r_{\uparrow \uparrow}}{\sqrt{2}}\left(\begin{array}{l}
1 \\
i \\
0 \\
0
\end{array}\right)+\frac{D}{\sqrt{2}}\left(\begin{array}{c}
0 \\
0 \\
1 \\
-1
\end{array}\right) e^{-\gamma_{0} x}
$$

for $x>0$, with $\gamma_{0}=V_{0} / v_{\mathrm{F}}$. The wave function in the pump remains unchanged. Substituting Eqs. (7) and (10) into Eq. (5), we obtain

$$
r_{\uparrow \uparrow}=i \frac{\cos \left(\varphi^{\prime} / 2\right)+i \cos (2 \phi) \sin \left(\varphi^{\prime} / 2\right)}{\cos \left(\varphi^{\prime} / 2\right)-i \cos (2 \phi) \sin \left(\varphi^{\prime} / 2\right)},
$$

with $\varphi^{\prime}=\varphi+\pi / 2$.

It is easy to see $\left|r_{\uparrow \uparrow}\right|^{2}=1$, essentially because the incident electron is fully reflected back into the spin-up channel. Moreover, for weak magnetic disorder, $\phi^{2} \ll 1, \arg \left(\mathrm{r}_{\uparrow \uparrow}\right)$ increments $2 \pi$ over a cycle. In other words, the trajectory of $r_{\uparrow \uparrow}$ forms a unit circle on the complex plane, indicating that the charge pumped for each cycle is $\Delta q=(-e)$, with $(-e)$ as the charge of an electron. Similarly, in the case of the spin-up channel being blocked, one can obtain $r_{\downarrow \downarrow}=\left.r_{\uparrow \uparrow}\right|_{\varphi \rightarrow-\varphi}$. On the complex plane, the trajectory of $r_{\downarrow \downarrow}$ still forms a unit circle, but $\arg \left(\mathrm{r}_{\downarrow \downarrow}\right)$ decrements $2 \pi$ over a cycle, so the charge pumped is $\Delta q=e$. To summarize, we have

$$
\Delta q=\mp e
$$

for the spin polarization of the lead parallel and antiparallel to the $z$ axis, respectively, as illustrated in Fig. 4. Since flipping the spin polarization of the lead does not change the topological (a) Spin-down channel blocked

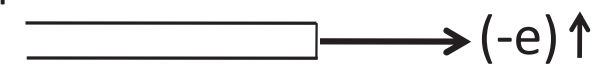

(b) Spin-up channel blocked

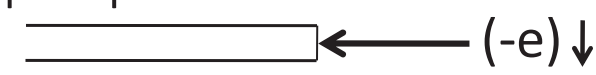

FIG. 4. Experimental detection of the spin Chern pumping effect. (a) When the spin polarization of the half-metallic lead is parallel to the $z$ axis, a quantized charge $(-e$ ) will be pumped into the lead per cycle. (b) If the spin polarization is flipped, a quantized charge $(-e)$ will be pumped in the opposite direction.

properties of the pump, this result vividly demonstrates that the pumping process in the present system is governed by "two" opposite Chern numbers that are inherent in the pump. The reversal of the sign of the quantized charge pumped per cycle with flipping the spin polarization of the half-metallic lead, if observed experimentally, can be considered as an unambiguous evidence for the existence of nonzero spin Chern numbers. Experimentally, the pumped charge can be obtained by measuring the electrical current. Once the electrical current $I$ is obtained, the pumped charge will be given by $\Delta q=$ $2 \pi I / \omega_{0}$.

\section{SUMMARY}

In summary, we have shown the principle of how a topological spin pump protected by spin Chern numbers can be built. The spin pumping effect in the spin Chern pump is robust against symmetry-breaking perturbations, in contrast to the $Z_{2}$ pump. A possible way to measure the spin Chern numbers experimentally was also proposed. While our work was based upon an abstract one-dimensional model mainly for simplicity of discussion, more realistic and easier-to-implement setups can be designed in the same spirit, which is beyond the main scope of this work.

\section{ACKNOWLEDGMENTS}

This work was supported by the State Key Program for Basic Research of China under Grants No. 2014CB921103 (L.S.), No. 2011CB922103, and No. 2010CB923400 (D.Y.X.); the National Natural Science Foundation of China under Grants No. 11225420 (L.S.), No. 11174125, and No. 91021003 (D.Y.X.); and a project funded by the PAPD of Jiangsu Higher Education Institutions. We also thank the U.S. NSF for support under Grants No. DMR-0906816 and No. DMR-1205734 (D.N.S.).
[1] K. Klitzing, G. Dorda, and M. Pepper, Phys. Rev. Lett. 45, 494 (1980).

[2] R. B. Laughlin, Phys. Rev. B 23, 5632 (1981).

[3] D. J. Thouless, M. Kohmoto, M. P. Nightingale, and M. den Nijs, Phys. Rev. Lett. 49, 405 (1982).

[4] D. J. Thouless, Phys. Rev. B 27, 6083 (1983).
[5] Q. Niu and D. J. Thouless, J. Phys. A 17, 2453 (1984).

[6] C. L. Kane and E. J. Mele, Phys. Rev. Lett. 95, 226801 (2005); B. A. Bernevig and S. C. Zhang, ibid. 96, 106802 (2006).

[7] J. E. Moore and L. Balents, Phys. Rev. B 75, 121306(R) (2007); L. Fu and C. L. Kane, ibid. 76, 045302 (2007); L. Fu, C. L. Kane and E. J. Mele, Phys. Rev. Lett. 98, 106803 (2007). 
[8] M. Z. Hasan and C. L. Kane, Rev. Mod. Phys. 82, 3045 (2010).

[9] X. L. Qi and S. C. Zhang, Physics Today 63, 33 (2010).

[10] F. D. M. Haldane, Phys. Rev. Lett. 61, 2015 (1988).

[11] C. L. Kane and E. J. Mele, Phys. Rev. Lett. 95, 146802 (2005).

[12] D. N. Sheng, Z. Y. Weng, L. Sheng, and F. D. M. Haldane, Phys. Rev. Lett. 97, 036808 (2006).

[13] E. Prodan, Phys. Rev. B 80, 125327 (2009); New J. Phys. 12, 065003 (2010).

[14] H. C. Li, L. Sheng, D. N. Sheng, and D. Y. Xing, Phys. Rev. B 82, 165104 (2010).

[15] Y. Yang, Z. Xu, L. Sheng, B. G. Wang, D. Y. Xing, and D. N. Sheng, Phys. Rev. Lett. 107, 066602 (2011).

[16] H. C. Li, L. Sheng, and D. Y. Xing, Phys. Rev. Lett. 108, 196806 (2012).

[17] H. C. Li, L. Sheng, R. Shen, L. B. Shao, B. G. Wang, D. N. Sheng, and D. Y. Xing, Phys. Rev. Lett. 110, 266802 (2013).

[18] L. Du, I. Knez, G. Sullivan, and R.-R. Du, arXiv:1306.1925.

[19] Xiao-Liang Qi, Taylor L. Hughes, and Shou-Cheng Zhang, Nat. Phys. 4, 273 (2008).

[20] F. Mahfouzi, B. K. Nikolic, S.-H. Chen, and C.-R. Chang, Phys. Rev. B 82, 195440 (2010).

[21] Y. Tserkovnyak, A. Brataas, G. E. W. Bauer, and B. I. Halperin, Rev. Mod. Phys. 77, 1375 (2005).

[22] O. Mosendz, J. E. Pearson, F. Y. Fradin, G. E. W. Bauer, S. D. Bader, and A. Hoffmann, Phys. Rev. Lett. 104, 046601 (2010).
[23] E. R. Mucciolo, C. Chamon, and C. M. Marcus, Phys. Rev. Lett. 89, 146802 (2002).

[24] T. Aono, Phys. Rev. B 67, 155303 (2003).

[25] R. Citro and F. Romeo, Phys. Rev. B 73, 233304 (2006).

[26] A. Schiller and A. Silva, Phys. Rev. B 77, 045330 (2008).

[27] C. W. Sandweg, Y. Kajiwara, A. V. Chumak, A. A. Serga, V. I. Vasyuchka, M. B. Jungfleisch, E. Saitoh, and B. Hillebrands, Phys. Rev. Lett. 106, 216601 (2011).

[28] M. Governale, F. Taddei, and R. Fazio, Phys. Rev. B 68, 155324 (2003).

[29] P. Sharma and C. Chamon, Phys. Rev. Lett. 87, 096401 (2001).

[30] R. Shindou, J. Phys. Soc. Jpn. 74, 1214 (2005).

[31] L. Fu and C. L. Kane, Phys. Rev. B 74, 195312 (2006).

[32] D. Meidan, T. Micklitz, and P. W. Brouwer, Phys. Rev. B 82, 161303 (2010).

[33] D. Meidan, T. Micklitz, and P. W. Brouwer, Phys. Rev. B 84, 195410 (2011).

[34] Fu and Kane studied the same model, focusing on the limit of weak coupling to leads [31]. We will consider the case of strong coupling, where the physics is found to be quite different.

[35] G. H. Wannier, Rev. Mod. Phys. 34, 645 (1962).

[36] X. L. Qi, Phys. Rev. Lett. 107, 126803 (2011).

[37] M. Büttiker, H. Thomas, and A. Prêtre, Z. Phys. B 94, 133 (1994).

[38] P. W. Brouwer, Phys. Rev. B 58, R10135 (1998). 